\title{
CD177 Gene
}

National Cancer Institute

\section{Source}

National Cancer Institute. CD177 Gene. NCI Thesaurus. Code C113573.

This gene is involved in platelet function. 